\section{The Hypoglycemic Effect of Intravenous Laser Therapy in Diabetic Mellitus Type 2 Patients; A Systematic Review and Meta-analyses}

\section{Abstract}

Objectives: Intravenous Laser Blood Irradiation (ILBI) is applied in some countries for the treatment of different diseases including diabetes mellitus. But there is not any systematic review available in this field. The aim of this study is to review the articles on the effects of ILIB in diabetic type 2 patients systematically.

Material and Methods: PubMed, SciELO, Science Direct and Google Scholar databases were searched for primary articles: The literature search was restricted to studies published in English and from the unrestricted past till April 2015. The keywords used were "Intravascular laser", "Intravenous laser", "low level laser", "low intensity laser", "phototherapy", "cold laser" combined with "fasting blood glucose", "blood sugar" and "blood glucose".

Results: Seven potentially suitable articles were found and among them four articles met all inclusion criteria. All these 4 articles were included in the final meta-analysis. Comparing before and after laser therapy, showed a significant decrease in glucose level (mean difference $=14.445,95 \% \mathrm{Cl}:-1.12$ to 30.031 $P=0.007)$. There was not any significant heterogeneity between trials $(P=0.158$, $12=42.3 \%)$.

Conclusion: ILIB is a safe and effective therapeutic modality for decreasing blood sugar level in diabetic type 2 patient.

Keywords: Intravenous laser; FBG; Systematic review

\section{Kazemilkhoo $\mathrm{N}^{1,2}$ \\ Ansari $F^{2}$ and \\ Nilforoushzadeh ${ }^{2}$}

Corresponding author:

Ansari F

Skin and Stem Cell Research Center, Tehran University of Medical Sciences, Tehran, Iran.

\section{fereshtehansari66@gmail.com}

Tel: $+98-21-61117000$

Received: September 28, 2015, Accepted: November 10, 2015, Published: November 13,2015

\section{Introduction}

During past 40 years, Low-level Laser Therapy (LLLT) has been broadly used in medical fields. Recently, there has been an increase in the clinical applications of low-level laser irradiation in various therapeutic fields. One of the most important functional aspects of laser therapy is photobiostimulatory effects of low-level lasers on various biological systems that is based on the effects of low intensity lasers, often described as lasers with less than 500 $\mathrm{mW}$ average power [1-4]. One of the Methods for irradiation of laser is Intravenous or (ILBI) with red, UV, and blue light, which is widely applied in the treatment of different pathologies. Blood irradiation therapy can be administered through a catheter in a vein, usually a vein in the forearm [5]. The medical effects are mainly systemic healing mechanisms including analgesic, biostimulative, immunocorrective, antibacterial, anti-allergic, antitoxic, vasodilative, antiarrhythmic, antihy-poxic, spasmolytic, anti-inflammatory, and other properties [6]. It expands the arteriovenous oxygen difference that affirms the liquidation of a tissue hypoxia and enrichment of oxygenation which is a sign of tissue metabolism normalization and improves oxidation of energycarrying molecules like glucose, pyruvate, and other substances. Laser irradiation activates ATP synthesis and energy formation in cells [5]. ILIB reduces glucose, cholesterol, low-density, and very low density lipoproteins (VLDL) and alleviates immune and hormonal system status. As ILBI has systematic effects, it can be a proper therapeutic modality in complex diseases like diabetes mellitus. Diabetes is a pathological metabolic state caused by 
insufficient transport and breakdown of glucose. It reveals low serum insulin to glucagon ratio and high levels of fatty acids. The liver produces glucose while other tissues use ketones and fatty acids instead of glucose [7].

As there is not any systematic review available in the field of the effects of ILIB in diabetic type 2 patients, we designed this study to review these articles systematically.

\section{Materials and Methods}

The following databases were searched for primary articles: PubMed, SciELO, Science Direct and Google Scholar. The literature search was restricted to studies published in English and from the unrestricted past till April 2015. The keywords used were "Intravascular laser", "Intravenous laser", "low level laser", "low intensity laser", "phototherapy", "cold laser" combined with "fasting blood glucose", "blood sugar" and "blood glucose".

Studies were eligible for inclusion if they fulfilled the following criteria: (i) enrolled participants diagnosed with type 2 diabetes; (ii) Intra venous laser irradiation was applied; (iii) The blood sugar of patients before and after laser irradiation has been measured.

Studies were excluded if they (i) included participants diagnosed with pre-diabetes, gestational diabetes, or type 1 diabetes; (ii) reported only categorical data of outcomes; or were posters or just abstracts. Studies that gave insufficient information regarding the laser interventions were also excluded if the related information could not be obtained from the corresponding authors.

Initial screen was based on titles or abstracts of retrieved publications; if they provided inadequate information with regard to inclusion or exclusion criteria, full-text articles were retrieved and evaluated. For each study, data regarding study sources (including author and publication year), characteristics of study population (including sample size, baseline mean age, sex [proportion of females] and duration of diabetes), characteristics of laser therapy (including frequency, intensity and wave length of laser applied), mean and standard deviation or standard error of mean of fasting blood sugar (FBS) before and after laser therapy and adherence and dropout rates, were extracted.

Two authors performed the literature selection, data collection, and quality assessment of studies.

In Kazemikhoo's [8] study, 2015 the results of blue and red laser irradiation and all the repetitions have been combined. The Change from baseline after laser irradiation was entered in the meta-analysis and SD of mean difference was calculated using SD of glucose level before and after laser therapy and $r$ equals 0.93 .

$r=\frac{S D_{B}^{2}+S D_{A}^{2}-S D_{C}^{2}}{2 \times S D_{B} \times S D_{A}}$

$r=$ Pearson correlation coefficient.

$\mathrm{SD}_{\mathrm{B}}=$ Standard deviation of glucose level before laser therapy.

$\mathrm{SD}_{\mathrm{B}}=$ Standard deviation of glucose level after laser therapy.

$\mathrm{SD}_{\mathrm{C}}=$ Standard deviation of glucose level changes.

The heterogeneity among studies was assessed using $Q$ test, with a $P$ value of $<10$ being considered of statistical significance.

Publication bias in the meta-analyses was detected and assessed by the Begg's test and Egger's test. Statistical analyses were performed using STATA Software (Version 12.0, College Station, Texas, USA).

\section{Results}

The initial search identified 7 potentially suitable articles and among them 4 articles met all inclusion criteria. All these 4 articles were included in the final meta-analysis.

The detailed characteristics of these trials are summarized in Table 1. Of these 4 trials, a total of 70 participants were included, with sample sizes ranging from 9 to 27 in individual trials.

All the participants were diabetic type 2 with the base line mean glucose level ranging from $190 \mathrm{mg} / \mathrm{dl}$ to $259 \mathrm{mg} / \mathrm{dl}$.

Table 1 Characteristics of included trials.

\begin{tabular}{|c|c|c|c|c|c|c|}
\hline Source & $\begin{array}{l}\text { Number of } \\
\text { subjects }\end{array}$ & $\begin{array}{l}\text { Mean age } \\
\text { (Years) }\end{array}$ & Sex(\%male) & Intervention & $\begin{array}{l}\text { Blood sugar } \\
\text { before } \mathrm{mg} / \mathrm{dl} \\
\text { (Mean } \pm \text { SD) }\end{array}$ & $\begin{array}{l}\text { Blood sugar after } \mathrm{mg} / \mathrm{dl} \\
\text { (Mean } \pm \text { SD) }\end{array}$ \\
\hline $\begin{array}{l}\text { R. Chen, } 2000 \text { (Chen, chen, Xie, } \\
\text { Chen, \& \& Zhang, 2000) }\end{array}$ & 10 & 67.3 & 93.3 & $\begin{array}{l}\text { He-Ne laser extravacular } \\
\text { irradiation therapy } \\
\text { instrument, , O-40mw, } \\
632.8 \mathrm{~nm}, 60 \mathrm{~min}\end{array}$ & $197.1 \pm 73.8$ & $106.2 \pm 540$ \\
\hline $\begin{array}{l}\text { T.V. Kovalyova, } 2002 \text { (Kovalyava, } \\
\text { 2002) }\end{array}$ & 27 & 57.3 & 13 & $\begin{array}{l}\text { ILBI intravenously } 2 \mathrm{~mW}, \mathrm{I} \\
=0,63 \mathrm{~mm} 405-\mathrm{nm} \mathrm{15}-30 \\
\mathrm{~min}\end{array}$ & $259.74 \pm 15.48$ & $255.78 \pm 15.3$ \\
\hline $\begin{array}{l}\text { N. KazemiKhoo, } 2013 \text { (N } \\
\text { Kazemi Khoo et al., 2013) }\end{array}$ & 9 & 60.63 & 55 & $\begin{array}{c}\text { ILBI intravenously } 1.5 \mathrm{~mW} \text {, } \\
\text { continuous, } 405-\mathrm{nm} 30 \\
\mathrm{~min}\end{array}$ & $190 \pm 17$ & $165 \pm 20$ \\
\hline $\begin{array}{l}\text { N. KazemiKhoo, } 2015 \\
\text { (KazemiKhoo \& Ansari, 2015) }\end{array}$ & 24 & 37 & 63.7 & $\begin{array}{l}\text { ILBI intravenously } 1.5 \mathrm{~mW} \text {, } \\
\text { continuous, } 405-\mathrm{nm} \text { and } \\
630 \mathrm{~nm} 20-30 \mathrm{~min}-\text { one } \\
\text { day blue one day red laser } \\
\text { therapy }\end{array}$ & $214.71 \pm 78.99$ & $188.53 \pm 75.60$ \\
\hline
\end{tabular}


Compared with the before laser therapy, the pooled estimate showed a significant decrease in glucose level (Mean difference $=14.445,95 \% \mathrm{Cl}:-1.12$ to $30.03, p=0.007$; Figure 1 ). There was not any significant heterogeneity between trials ( $P=0.158,12=42.3 \%)$.

There was no significant publication bias as evidenced by the Begg's test and Egger's test (all $P>05$ ).

\section{Discussion}

The result of this meta-analysis suggests that Intravenous laser therapy decreases blood glucose level in diabetic type 2 patients significantly. Immediately after ILIB blood glucose decreases $14 \mathrm{mgr} / \mathrm{dlit}$. It seems that laser irradiation may have an effect on arginine and increase nitric oxide (NO) production. Arginine affects on the release of hormone like glucagon, insulin, prolactin, adrenal catecholamins and growth hormone [9]. It decreases tissue hypoxia, stimulates oxygenation and normalizes tissue metabolism [10]. Ramadawon concluded that even in advanced cases of diabetes mellitus, laser therapy could restore the pancreas function and normalize the blood glucose level. They concluded that laser irradiation could stimulate and regenerate pancreatic tissues, including the Langerhans $\beta$-cells, even in advanced disease states [11].Tiedan et al reported that ATPase was significantly lower in diabetic patients than that in control subjects, and laser irradiation significantly activated $\mathrm{Na}^{+} / \mathrm{K}^{+}-$ ATPase, $\mathrm{Ca}^{2+}, \mathrm{Mg}^{2+}$-ATPase. They suggested that intravascular laser might be a new complex therapeutics for diabetes [12].

However, it shows inadequate evidence regarding the effects of other factors related to health of diabetic people. Numerous studies showed the positive effect of laser therapy on diabetic complications [13-17]. Our previous studies showed that laser irradiation may be effective in neuropathy and wound healing in these patients [18-20].It could be recommended to include these factors for future systematic reviews.

Since results from this meta-regression find intravenous laser therapy as an effective way to decrease blood sugar level in diabetic patients it could be suggested to prescribe this kind of therapy for patients with type 2 diabetes along with other classic methods such as diet, weight control, medications and exercise to gain benefits on glycemic control $[21,22]$.

To the time being, this is the first analysis that systematically assesses the hypoglycemic effect of intravenous laser therapy, in patients with type 2 diabetes. This study analyses the quantity of therapeutic effects of this method including Mean, SD and SE. We included trials with before-after study designs. Even though it is difficult to assess causal association in this kind of trials, they are the most feasible way to measure medical intervention effects. Due to small numbers of trials in this field it was the only way to include more trials and to improve external validity of the meta-analysis.

It is recommended that future RCTs with proper control groups and appropriate description of adequate information should be conducted. Despite no significant publication bias was detected by the Begg's test and Egger's test, the risk of publication bias still cannot be fully ruled out due to the language restriction to English, the selection of only published papers, as well as the potentially underpowered statistical tests.

In conclusion, the meta-analysis shows that Intravenous laser therapy is associated with a decreased blood sugar level among

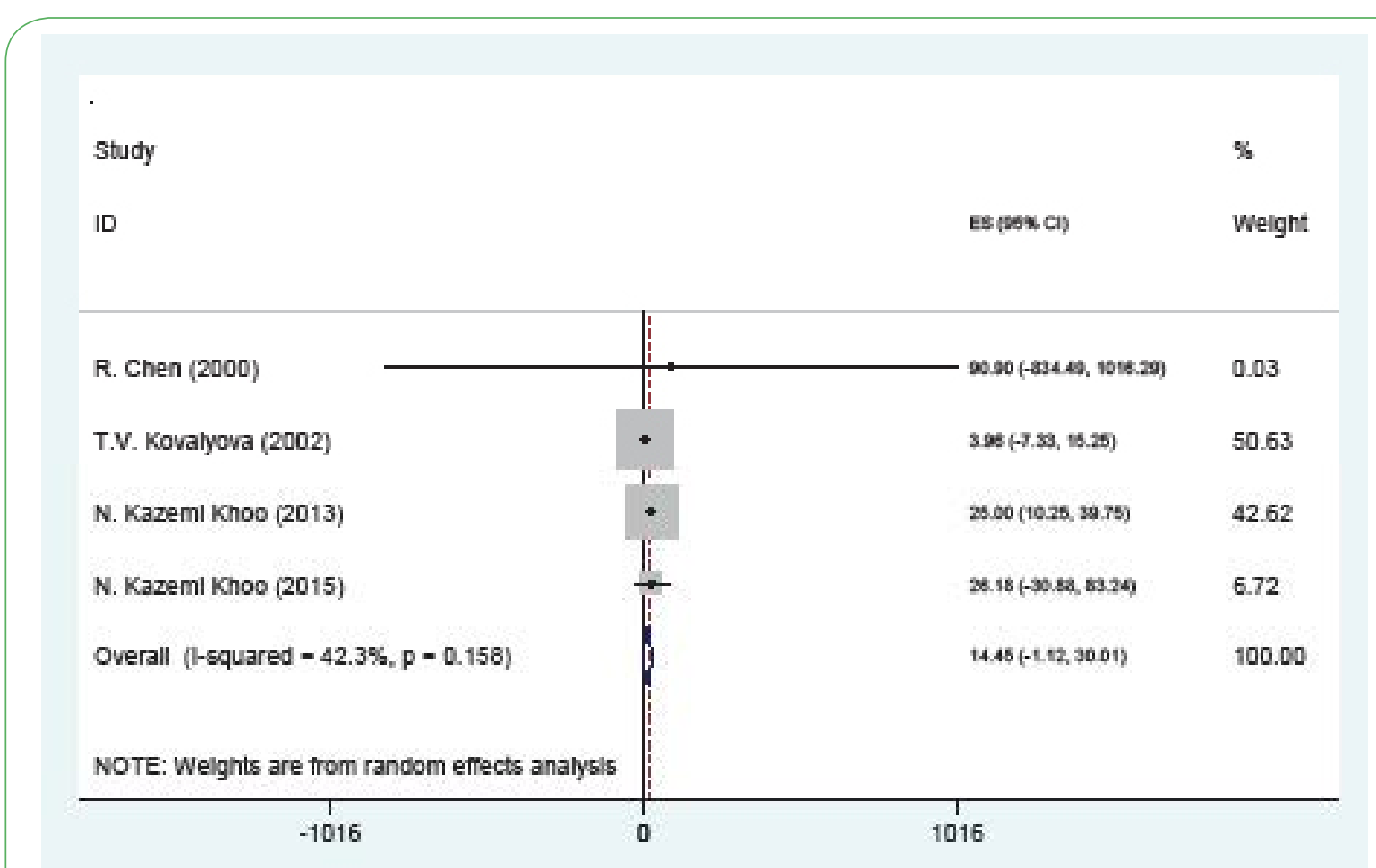

Figure 1 Forest plot of trials examining LLLT effects on glucose level in type 2 diabetes patients. ES, mean difference; $\mathrm{Cl}$, confidence interval Summary estimates were analyzed with a random-effects model. 
patients with type 2 diabetes. As the selected studies evaluate the effects of intravenous laser for a short period of time, it seems more studies are needed to assess the long term effects. Future RCTs with head-to-head designs comparing intravenous laser therapy versus hyperglycemia control drugs, and with extended length of interventions ( 6 months) and with assessing more health related outcomes, are required to strengthen the findings in this meta-analysis.

\section{Acknowledgements}

We dedicate here our acknowledgments to Dr.Mohammadreza Rahbar for English Edition of the manuscript. 


\section{References}

1 Grossman N, Schneid N, Reuveni H, Halevy S, Lubart R (1998) 780 $\mathrm{nm}$ low power diode laser irradiation stimulates proliferation of keratinocyte cultures: involvement of reactive oxygen species, Lasers in surgery and medicine 22: 212-218.

2 Lubart R, Wollman Y, Friedmann H, Rochkind S, Laulicht I (1992) Effects of visible and near-infrared lasers on cell cultures. J Photochem Photobiol B 12: 305-310.

3 Passarella S, Casamassima E, Molinari S, Pastore D, Quagliariello E, et al. (1984) Increase of proton electrochemical potential and ATP synthesis in rat liver mitochondria irradiated in vitro by helium-neon laser. FEBS letters 175: 95-99.

4 Yu W, Naim J, Lanzafame R (1994) The effects of photo-irradiation on the secretion of TGF and PDGF from fibroblasts in vitro. Lasers Surg Med Suppl 6.

5 Gasparyan L (2003) Laser Irradiation of the blood. Laser PartnerClinixperience 1-4.

6 Moshkovska T, Mayberry J (2005) It is time to test low level laser therapy in Great Britain. Postgrad Med J 81: 436-441.

7 Makela A (2004) Theoretical backgrounds for light application in diabetes, Laser, Florence.

8 KazemiKhoo N, Ansari F (2015) Blue or red: which intravascular laser light has more effects in diabetic patients? Lasers Med Sci 30: 363366.

9 Makela A (2005) Role of L-arginine in the biological effects of blue light [5968-06]. Proceedings-Spie The International Society For Optical Engineering.

10 Brill G, Gasparyan L, Makela A (2003) Modification in relationships in the system endothelial cell-blood platelet by low level laser radiation. Laser Florence meeting.

11 Ramdawon P (1999) Bioresonance information laser therapy of diabetes mellituse a first clinical experience of the hypoglycemic effect of low level laser terapy and its perspectives, Paper presented at the North American Laser Therapy Association Conference.
12 Tiedan W (1992) Effects of low-energy He-Ne laser irradiation of extracorporeally circulatory blood on ATPase activities of erythrocyte membrane in patients with IDDM [J]. Laser Journal 6: 011.

13 Chung H, Dai T, Sharma SK, Huang YY, Carroll JD, et al. (2012) The nuts and bolts of low-level laser (light) therapy. Ann Biomed Eng 40: 516-533.

14 Hawkins D, Houreld N, Abrahamse H (2005) Low level laser therapy (LLLT) as an effective therapeutic modality for delayed wound healing. Ann N Y Acad Sci 1056: 486-493.

15 Maiya GA, Kumar P, Rao L (2005) Effect of low intensity heliumneon (He-Ne) laser irradiation on diabetic wound healing dynamics. Photomed Laser Surg 23: 187-190.

16 Schindl A, Schindl M, Schön H, Knobler R, Havelec L, et al. (1998) Low-intensity laser irradiation improves skin circulation in patients with diabetic microangiopathy. Diabetes Care 21: 580-584.

17 Zinman LH, Ngo M, Ng ET, Nwe KT, Gogov S, et al. (2004) Lowintensity laser therapy for painful symptoms of diabetic sensorimotor polyneuropathy: a controlled trial. Diabetes Care 27: 921-924.

18 Kazemi-Khoo N (2006) Successful treatment of diabetic foot ulcers with low-level laser therapy. The Foot 16: 184-187.

19 Khamseh ME, Kazemikho N, Aghili R, Forough B, Lajevardi M, et al. (2011) Diabetic distal symmetric polyneuropathy: effect of lowintensity laser therapy. Lasers Med Sci 26: 831-835.

20 Kazemi-Khoo N, Shokrgozar MA, Kashani IR, Amanzadeh A, Mostafavi E, et al. (2014) In vitro therapeutic effects of low level laser at mRNA level on the release of skin growth factors from fibroblasts in diabetic mice. Avicenna journal of medical biotechnology 6: 113 .

21 DeFronzo RA, Ratner RE, Han J, Kim DD, Fineman MS, et al. (2005) Effects of exenatide (exendin-4) on glycemic control and weight over 30 weeks in metformin-treated patients with type 2 diabetes. Diabetes Care 28: 1092-1100.

22 Turner RC, Cull CA, Frighi V, Holman RR (1999) Glycemic control with diet, sulfonylurea, metformin, or insulin in patients with type 2 diabetes mellitus: progressive requirement for multiple therapies (UKPDS 49). UK Prospective Diabetes Study (UKPDS) Group. JAMA 281: 2005-2012. 